\title{
Short-Time Fourier Transform: Two Fundamental Properties and an Optimal Implementation
}

\author{
Lütfiye Durak, Student Member, IEEE, and Orhan Arıkan, Member, IEEE
}

\begin{abstract}
Shift and rotation invariance properties of linear time-frequency representations are investigated. It is shown that among all linear time-frequency representations, only the short-time Fourier transform (STFT) family with the Hermite-Gaussian kernels satisfies both the shift invariance and rotation invariance properties that are satisfied by the Wigner distribution (WD). By extending the time-bandwidth product (TBP) concept to fractional Fourier domains, a generalized time-bandwidth product (GTBP) is defined. For mono-component signals, it is shown that GTBP provides a rotation independent measure of compactness. Similar to the TBP optimal STFT, the GTBP optimal STFT that causes the least amount of increase in the GTBP of the signal is obtained. Finally, a linear canonical decomposition of the obtained GTBP optimal STFT analysis is presented to identify its relation to the rotationally invariant STFT.
\end{abstract}

Index Terms-Fractional Fourier transform, generalized timebandwidth product, linear time-frequency representations, rotation invariance, short-time Fourier transform.

\section{INTRODUCTION}

$\mathbf{R}$ ESEARCH on time-frequency domain characterization of signals has been focused on the variants of short-time Fourier transform (STFT) [1]-[7] and Wigner distribution (WD) [8], [9]. The absence of undesirable cross terms [1], [10] and computational simplicity [11]-[13] are the major factors in the wide-spread use of the STFT in practice. With the advance of faster processors, the efficiency of the STFT techniques have become less important. However, the ability of representing time-frequency content of signals free of cross terms is still the major advantage of the STFT techniques over the WD-related quadratic time-frequency distributions.

Among its many important properties, the STFT has a fundamental property that simplifies the interpretation of the resultant distribution: magnitude-wise shift invariance in both time and frequency. In this paper, we first prove that the STFT is the only linear distribution that has the magnitude-wise shift invariance property in both time and frequency. Then, we investigate time-frequency domain rotation property within the general class of linear distributions. This lesser known property, which is satisfied by the WD, is defined as follows: A time-frequency distribution satisfies the rotation property if the distribution of an arbitrary signal and the distribution of its $a$ th-order fractional

Manuscript received August 28, 2001; revised October 23, 2002. The associate editor coordinating the review of this paper and approving it for publication was Prof. Paulo S. R. Diniz.

The authors are with the Department of Electrical Engineering, Bilkent University, Ankara, Turkey (e-mail: lutfiye@ee.bilkent.edu.tr; oarikan@ee.bilkent.edu.tr).

Digital Object Identifier 10.1109/TSP.2003.810293
Fourier transformation are $a \pi / 2$ rad rotated versions of each other [14], [15]. We start our investigation on linear time-frequency distributions by showing that STFT satisfies the rotation property only if the STFT kernel is a Hermite-Gaussian function. Thus, we reach the conclusion that the linear time-frequency distributions, which satisfy both the rotation property and the magnitude-wise time and frequency shift property, are the STFT with Hermite-Gaussian kernels.

The choice of the STFT kernel determines the time-frequency signal localization properties of the distribution. Among the Hermite-Gaussian function family, since it has the minimum time-bandwidth product (TBP), the Gaussian function is the most commonly used kernel function. However, STFT with the Gaussian kernel still suffers from the problem of limited resolution. To overcome the inherent tradeoff between the time and the frequency localization of the STFT, several alternatives have been investigated in the literature. In [5], using two kernel functions of different supports, a wideband and a narrowband spectrogram are obtained. In order to preserve the localization characteristics of both, a combined spectrogram is formed by computing the geometric mean of the corresponding STFT magnitudes, whereas in [6], the STFT is evaluated by using a kernel function with an adaptive width in order to analyze the transient response of radar targets. In [16], a kernel matching algorithm is developed by locally adapting the Gaussian kernel functions to the analyzed signal. Although these investigations provide significant improvements in the time-frequency localization of signal components, in the presence of chirp-like signals, they still provide descriptions whose localization properties depends on the chirp rate of the components. Recently, [7] introduced an improved instantaneous frequency estimation technique using an adaptive STFT where the kernel functions are chosen from a set of functions through adaptation rules and computation of the STFT with varying kernel functions at each time instance. In addition, apart from the analysis of deterministic signals, there have been studies where time-varying spectra of random processes are investigated [17].

In this paper, we characterize the time-frequency domain localization by STFT and investigate the effect of the STFT kernel on the obtained time-frequency representation of signals. We introduce the generalized time-bandwidth product (GTBP) definition to provide a rotation-invariant measure of signal support in the time-frequency domain. Then, we obtain the optimal STFT kernel that provides the most compact representation considering the GTBP of a signal component. The proposed time-frequency analysis is shown to be equivalent to an ordinary STFT analysis conducted in a scaled fractional Fourier transform domain. The obtained GTBP optimal STFT representation yields 
optimally compact time-frequency supports for chirp-like signals on the STFT plane. In general, the GTBP optimal STFT representation does not satisfy the rotation property. However, as shown in detail, there exists a linear canonical decomposition of the GTBP optimal STFT that provides the link between the GTBP optimal STFT and the rotation invariance property.

This paper is organized as follows. In Section II, we show that the STFT is the only linear time-frequency representation that satisfies the magnitude-wise shift invariance property. In Section III, we define the rotation property of shift-invariant time-frequency distributions and obtain the class of STFT kernels satisfying the rotation property. In Section IV, we introduce the GTBP and obtain the GTBP optimal STFT kernel. In Section $\mathrm{V}$, we present a linear canonical decomposition of the GTBP optimal STFT and relate it to the rotation property. Furthermore, the performance of the GTBP optimal STFT representation is illustrated by using simulated data. Finally, the paper is concluded in Section VI with future research directions.

\section{LINEAR SHIFT-INVARIANT TIME-FREQUENCY DISTRIBUTIONS}

Time-frequency distributions are designed to characterize the time-frequency content of signals. Since time or frequency shifts do not change the time-frequency content of a signal, except to relocate it correspondingly, it is important that time-frequency representations satisfy the magnitude-wise shift invariance property. A precise statement of this property is given as follows: A time-frequency representation $D_{x}(t, f)$ is magnitude-wise shift invariant if for $x_{s}(t)=x\left(t-t_{s}\right) \cdot e^{j 2 \pi f_{s} t}$

$$
\left|D_{x_{s}}(t, f)\right|=\left|D_{x}\left(t-t_{s}, f-f_{s}\right)\right|, \forall x(t), t_{s}, f_{s} .
$$

In this section, we investigate the magnitude-wise shift invariance property within the class of linear time-frequency representations.

The magnitude-wise shift invariance of linear time-frequency distributions can be characterized fully as follows. The general kernel-based form of a linear time-frequency distribution $D_{x}(t, f)$ is given by ${ }^{1}$

$$
D_{x}(t, f)=\int \kappa\left(t, f, t^{\prime}\right) x\left(t^{\prime}\right) d t^{\prime}
$$

where $\kappa\left(t, f, t^{\prime}\right)$ is the kernel of the distribution [18]. By making use of the general theorem on linear systems given in Appendix A, it can be shown easily that the magnitude-wise shift invariance in time requires $D_{x}(t, f)$ to have the following form:

$$
D_{x}(t, f)=e^{\widehat{\jmath \phi(}(t, f)} \int \kappa\left(t-t^{\prime}, f\right) x\left(t^{\prime}\right) d t^{\prime} .
$$

Since the magnitude of time-frequency distributions are related to the energy distribution of the signals in the time-frequency plane, $e^{\widehat{\jmath \phi}(t, f)}$ will be ignored in the rest of the derivations. The

\footnotetext{
${ }^{1}$ All integrals are from $-\infty$ to $+\infty$ unless otherwise stated.
}

implications of magnitude-wise shift invariance in frequency can be investigated in the Fourier domain as

$$
\begin{aligned}
D_{x}(t, f) & =\int \kappa\left(t-t^{\prime}, f\right) \int X\left(f^{\prime}\right) e^{\jmath 2 \pi f^{\prime} t^{\prime}} d f^{\prime} d t^{\prime} \\
& =e^{\jmath 2 \pi f t} \int \Gamma\left(f, f^{\prime}\right) e^{-\jmath 2 \pi\left(f-f^{\prime}\right) t} X\left(f^{\prime}\right) d f^{\prime}
\end{aligned}
$$

where $X(f)$ is the Fourier transform of $x(t)$, and $\Gamma\left(f, f^{\prime}\right)=\int \kappa\left(t^{\prime \prime}, f\right) e^{-\jmath 2 \pi f^{\prime} t^{\prime \prime}} d t^{\prime \prime}$. The magnitude-wise shift invariance with respect to frequency requires that

$$
\Gamma\left(f, f^{\prime}\right)=G\left(f-f^{\prime}\right) e^{j \tilde{\phi}(f)} .
$$

Thus, the kernel has the following representation:

$$
\begin{aligned}
\kappa(t, f) & =e^{\tilde{\phi}(f)} \int G\left(f-f^{\prime}\right) e^{\jmath 2 \pi f^{\prime} t} d f^{\prime} \\
& =g(-t) e^{\jmath 2 \pi f t} e^{\jmath \tilde{\phi}(f)} \cdot
\end{aligned}
$$

Since the phase $e^{\jmath 2 \pi f t}$ and $e^{\jmath \tilde{\phi}(f)}$ can be ignored, the general form of a magnitude-wise shift invariant linear time-frequency distribution is

$$
D_{x}(t, f)=\int g\left(t^{\prime}-t\right) x\left(t^{\prime}\right) e^{-\jmath 2 \pi f t^{\prime}} d t^{\prime}
$$

which has the same form of the STFT [1], [3] with the kernel $g(t)$. Consequently, STFT is the only distribution that is linear and magnitude-wise shift-invariant under both time and frequency shifts.

\section{LINEAR TIME-FREQUENCY DISTRIBUTIONS AND THE ROTATION PROPERTY}

The lesser known rotation property of time-frequency distributions is defined as follows:

Definition 1: A time-frequency distribution $D_{x}(t, f)$ satisfies the rotation property [19] if for all $x(t)$ and $a$

$$
\left|D_{x_{a}}(t, f)\right|=\left|R_{-\phi}\left\{D_{x}(t, f)\right\}\right|
$$

where $\phi$ is equal to $a \pi / 2$, and $x_{a}$ is the $a$ th-order fractional Fourier transformation (FrFT) of $x(t)$ given by [20], [21]

$$
\begin{aligned}
x_{a}(t) & \equiv\left\{\mathcal{F}^{a} x\right\}(t) \\
& =\int B_{a}\left(t, t^{\prime}\right) x\left(t^{\prime}\right) d t^{\prime}, \quad a \in \Re, 0<|a|<2
\end{aligned}
$$

where $B_{a}\left(t, t^{\prime}\right)$ is

$B_{a}\left(t, t^{\prime}\right)=\frac{e^{-j(\pi \operatorname{sgn}(\sin \phi) / 4+\phi / 2)}}{|\sin \phi|^{1 / 2}} e^{j \pi\left(t^{2} \cot \phi-2 t t^{\prime} \csc \phi+t^{\prime 2} \cot \phi\right)}$

and the rotation operator acting on a two-dimensional (2-D) function $D(u, v)$ is defined as

$$
R_{\phi}\{D(u, v)\}=D(u \cos \phi+v \sin \phi,-u \sin \phi+v \cos \phi) \text {. }
$$

It has been shown that a quadratic time-frequency distribution satisfies the rotation property only if it has a rotationally 
symmetric kernel [22]. The most widely known quadratic distribution satisfying the rotation property is the WD

$$
W_{x_{a}}(t, f)=R_{-\phi}\left\{W_{x}(t, f)\right\}, \quad \phi=\frac{a \pi}{2}
$$

where $W_{x}$ is the WD of $x(t)$ [14], [15], [19]. As the time and frequency variables have different units, a dimensional normalization of the time-frequency plane is required before performing the rotation operation [23]. It is assumed that the time and frequency domain representations of a signal are confined to $[-\Delta t / 2, \Delta t / 2]$ and $[-\Delta f / 2, \Delta f / 2]$ intervals, respectively. Then, a scaling parameter $s$ is introduced, where the dimension of time and scaled coordinates $t / s$ and $f s$ are used as new coordinates. This way, the time and frequency representations of the signal are confined to intervals of length $\Delta t / s$ and $\Delta f s$. We choose $s=\sqrt{\Delta t / \Delta f}$ so that the lengths of both intervals are equal to $\sqrt{\Delta t \Delta f}$, which is a dimensionless quantity. In numerical examples, signals can be represented in both domains with $N=\Delta t \Delta f$ samples spaced $1 / \sqrt{N}$ apart. In the paper, we assume that a dimensional normalization has already been done and all the coordinates represent dimensionless quantities.

The rotation property has some important conceptual and practical implications [14], [15], [24]-[27]. It implies that the inherent time-frequency domain characteristics of a signal remains unchanged in all the fractional Fourier domains. Therefore, there is nothing special about any of the fractional Fourier domain representations of a signal including the commonly used time or frequency domains. Among the linear, shift-invariant time-frequency distributions, only the STFT with Hermite-Gaussian kernels satisfies the rotation property. This can be shown as follows:

Proposition 1: STFT satisfies the rotation property only if the window function is a Hermite-Gaussian function.

Proof: The STFT of the fractionally Fourier transformed $x(t)$ is

$$
\operatorname{STFT}_{x_{a}}(t, f)=\int x_{a}\left(t^{\prime}\right) h^{*}\left(t^{\prime}-t\right) e^{-\jmath 2 \pi f t^{\prime}} d t^{\prime} .
$$

By using (9) and the property $B_{a}\left(t^{\prime}, \tau\right)=B_{-a}^{*}\left(t^{\prime}, \tau\right)[21]$, we obtain

$$
\begin{aligned}
\operatorname{STFT}_{x_{a}}(t, f)= & \int x(\tau) \\
& \cdot\left[\int h\left(t^{\prime}-t\right) e^{\jmath 2 \pi f t^{\prime}} B_{-a}\left(t^{\prime}, \tau\right) d t^{\prime}\right]^{*} d \tau \\
= & e^{-\jmath \pi \theta} \int x(\tau) h_{-a}^{*}(\tau-t \cos \phi-f \sin \phi) \\
& \cdot e^{-\jmath 2 \pi \tau(f \cos \phi-t \sin \phi)} d \tau
\end{aligned}
$$

where $\theta=\sin \phi \cos \phi\left(t^{2}-f^{2}\right)+2 f t \sin ^{2} \phi$. The rotation property requires that the magnitude of (13) is equal to the magnitude of $R_{\phi}\left\{\operatorname{STFT}_{x}(t, f)\right\}$, which imposes the following condition on $h(t)$ to hold true for all $\phi$

$$
h(u)=h_{-a}(u) \cdot e^{-\jmath \alpha}
$$

where $\alpha$ does not depend on $u$ but may be a function of $a$. The equality in (15) can only be satisfied if $h(t)$ is one of the eigenfunctions of the FrFT operator. The Hermite-Gaussian functions form the complete set of eigenfunctions of the FrFT operator defined in (9) [21]. Then, $e^{-\jmath \alpha}$ will be the eigenvalue of the FrFT such that $\alpha$ equals $(\pi / 2) a l$, where $l$ is the Hermite polynomial order.

The rotation property of the STFT with the zeroth-order Hermite-Gaussian kernel is illustrated in Fig. 1(c) and (d), which corresponds to the STFT of the time domain signal and the 0.5 th-order fractionally Fourier transformed signal in Fig. 1(a) and (b), respectively. The rotation property fails if the STFT of both of these signals are computed with a scaled Gaussian window function, as shown in Fig. 1(e) and (f) .

It will be shown next that the rotation property fails when the STFT kernel is a linear combination of many Hermite-Gaussian functions.

Proposition 2: The rotation property fails when the STFT kernel is a linear combination of Hermite-Gaussian functions.

Proof: The Hermite-Gaussian functions are the eigenfunctions of the FrFT. For each FrFT order $a$

$$
\left\{\mathcal{F}^{a} h_{l}\right\}(t)=\lambda_{l}^{a} h_{l}(t)
$$

where $h_{l}(t)$ is the $l$ th-order Hermite-Gaussian function, and $\lambda_{l}=e^{-\jmath l \pi / 2}$. If $h(u)$ is a combination of Hermite-Gaussian functions as $h(u)=\sum_{k=1}^{M} \alpha_{k} h_{k}(u)$ with arbitrary nonzero $\alpha_{k}$ coefficients, then $h_{-a}(u)$ on the left-hand side of (15) is

$$
\begin{aligned}
h_{-a}(u) & =\left\{\mathcal{F}^{-a}\left(\sum_{k=1}^{M} \alpha_{k} h_{k}\right)\right\}(u) \\
& =\sum_{k=1}^{M} \alpha_{k} \lambda_{k}^{-a} h_{k}(u) .
\end{aligned}
$$

The condition in (15) requires (17) be equal to $e^{-\jmath \alpha} \sum_{k=1}^{M} \alpha_{k} h_{k}(u)$. However, for arbitrary $a$, if $i \neq j$, then $\lambda_{i}^{-a} \neq \lambda_{j}^{-a}$. Therefore, any linear combination of Hermite-Gaussian functions fails to satisfy the rotation property of STFT.

\section{TIME-FREQUENCY DOMAIN LOCALIZATION BY STFT}

An important criterion for the success of time-frequency representations is how well it preserves the time-frequency domain support of signals. Among the commonly used time-frequency representations, WD is the best in this respect. However, the cross-terms of the WD clutters the obtained time-frequency representation. Therefore, in a way, it disturbs the actual support of the signal in the time-frequency domain. The STFT family provides cross-term free time-frequency representations. So, support preservation criteria is applicable to measure the success of the alternative STFT representations. In this section, we investigate the effect of the STFT kernel on the obtained time-frequency support of the signal components. This investigation will require generalized definition of the time-bandwidth product. Furthermore, it will lead to signal adaptive STFTs. 

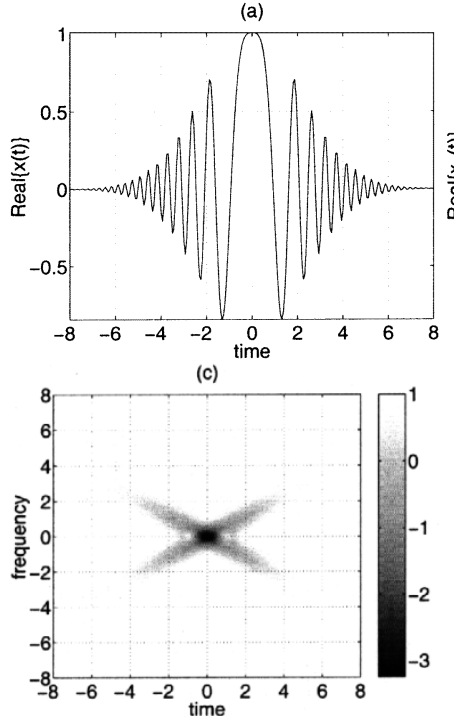

(e)
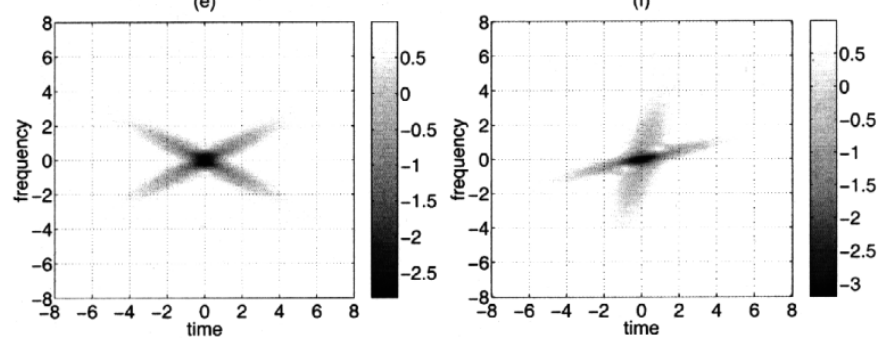

Fig. 1. STFT satisfies the rotation property only with Hermite-Gaussian kernels. To illustrate this, a two-component chirp signal is used. (a) Real part of the signal. (b) Real part of the $a=0.5$ th-order FrFT of the signal. (c) STFT of the signal. (d) STFT of its $a=0.5$ th-order FrFT with zeroth-order Hermite-Gaussian kernel. The STFT of the transformed signal is the rotated version of the original STFT shown in (c). However, as shown in (e) and (f), the STFT fails to satisfy the rotation property if its kernel is not a Hermite-Gaussian function. (e) STFT of the signal. (f) STFT of the fractionally Fourier transformed signal with the kernel $h(t)=e^{-\pi t^{2} / 3}$.

The time-frequency domain support of a signal $x(t)$ is commonly measured by its time-width $T_{x}$ and its frequency domain bandwidth $B_{x}$, which are defined as

$$
\begin{aligned}
& T_{x}=\frac{\left[\int\left(t-\eta_{t}\right)^{2}|x(t)|^{2} d t\right]^{1 / 2}}{\|x\|} \\
& B_{x}=\frac{\left[\int\left(f-\eta_{f}\right)^{2}|X(f)|^{2} d f\right]^{1 / 2}}{\|x\|}
\end{aligned}
$$

where

$$
\begin{aligned}
\eta_{t} & =\frac{\left[\int t|x(t)|^{2} d t\right]}{\|x\|^{2}} \\
\eta_{f} & =\frac{\left[\int f|X(f)|^{2} d f\right]}{\|x\|^{2}}
\end{aligned}
$$

for a Fourier transform pair $x(t)$ and $X(f)$. Therefore, the TBP, which is defined as

$$
\operatorname{TBP}\{x(t)\}=T_{x} \cdot B_{x}
$$

has been commonly used as a measure for the time-frequency domain support of the signal. The well-known uncertainty principle dictates that $1 /(4 \pi)$ is a lower bound on the TBP of a signal, and only the Gaussian signal has a TBP equal to this lower bound [21].

If the TBP is chosen as the measure of support, a well-defined optimization problem can be cast for the optimal STFT kernel as follows. As shown in Fig. 2(a), for an STFT kernel $g(t)$, the time-frequency domain support of the representation for $x(t)$ can be zoned into a rectangular region of respective time-frequency dimensions of $\left(T_{x}^{2}+T_{g}^{2}\right)^{1 / 2}$ and $\left(B_{x}^{2}+B_{g}^{2}\right)^{1 / 2}$ [3]. To choose the optimal window that adapts to the analyzed signal $x(t)$, the following optimization criterion can be used:

$$
\min _{T_{g}, B_{g} ; T_{g} \cdot B_{g} \geq(1 / 4 \pi)}\left(T_{x}^{2}+T_{g}^{2}\right)^{1 / 2} \cdot\left(B_{x}^{2}+B_{g}^{2}\right)^{1 / 2} .
$$

It can be easily seen that the optimal solution $g(t)$ must satisfy the uncertainty principle with equality; therefore, it must be a Gaussian kernel. Hence, the optimization problem can be solved in this set by just obtaining the time-width of the Gaussian by solving the following problem:

$$
\min _{T_{g}}\left(T_{x}^{2}+T_{g}^{2}\right)^{1 / 2} \cdot\left(B_{x}^{2}+\frac{1}{16 \pi^{2}} \frac{1}{T_{g}^{2}}\right)^{1 / 2} .
$$

With a little effort the optimal $T_{g}$ can be obtained as

$$
T_{g}=\sqrt{\frac{T_{x}}{4 \pi B_{x}}}
$$

and the corresponding optimal Gaussian window is [3]

$$
g_{T B P}(t)=e^{-\pi t^{2} B_{x} / T_{x}}
$$

For $x(t)$, itself a Gaussian signal with $T_{x} \cdot B_{x}=1 / 4 \pi$, this optimal time-support for the Gaussian kernel function reduces to the commonly used rule-of-thumb of choosing the $T_{g}=T_{x}$. Otherwise, the optimal time support $T_{g}$ is always shorter than the time support of the analyzed signal $T_{x}$.

Although the TBP of a signal is commonly used, it is not a satisfactory measure for the time-frequency support of signals. This is illustrated in Fig. 2(c), where support of a signal and its bounding rectangle of sides time and frequency widths and area equal to TBP is shown. Even though the rotation operation just rotates the support of $x(t)$, the TBP changes. In Fig. 2(b), the dependence of the TBP on the FrFT order $a$ is shown for a scaled Gaussian signal. The minimum TBP is reached when the signal support lies along the time or frequency axes. As seen from this example, TBP is always an upper bound to the support of the signal in the time-frequency domain. Therefore, there is a need for a tighter measure of the support. Here, we propose a new measure that we call generalized TBP, which is defined as

$$
\operatorname{GTBP}\{x(t)\}=\min _{0 \leq a<4} \operatorname{TBP}\left\{x_{a}(t)\right\}
$$

As illustrated in Fig. 2(d), the GTBP provides the tightest bounding rectangle to the support of the signal in the time-frequency domain, hence providing a more representative support information than the TBP. As the well known lower bound on the TBP, a lower bound for the GTPB also exists, and they are equal to each other. A more detailed discussion of the uncertainty relationship in the fractional Fourier domains can be found in [28]-[31]. 


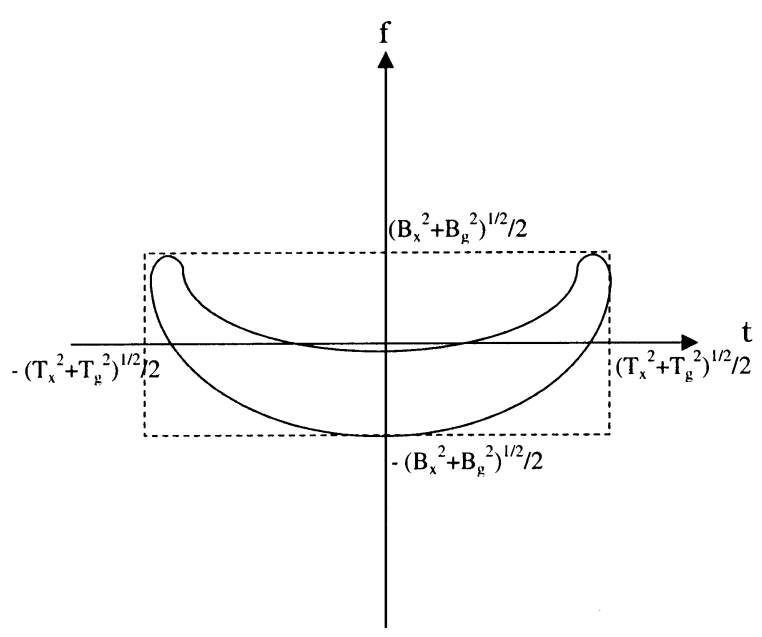

(a)

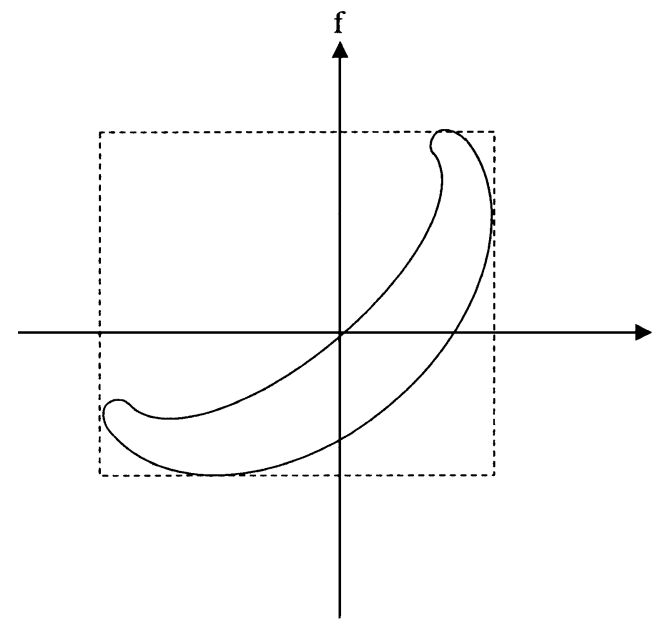

(c)

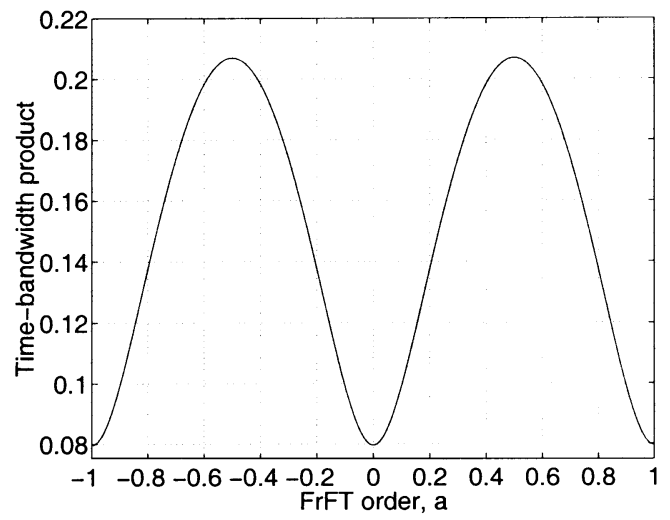

(b)

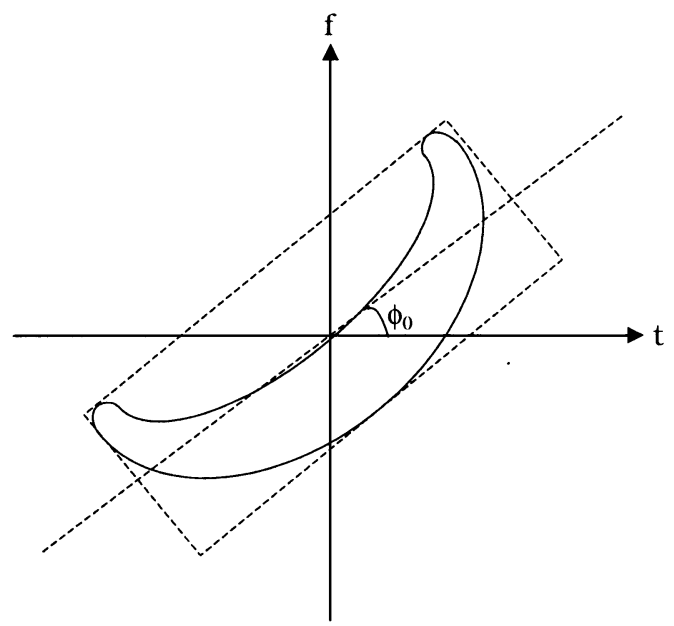

(d)

Fig. 2. Time-frequency domain support of the STFT representation for $x(t)$ with kernel $g(t)$ can be zoned into a rectangular region of respective time-frequency dimensions of $\left(T_{x}^{2}+T_{g}^{2}\right)^{1 / 2}$ and $\left(B_{x}^{2}+B_{g}^{2}\right)^{1 / 2}$ as shown in (a). Even though the rotation operation does not change the area of the support of a signal, the TBP changes. (b) Dependence of the TBP on the FrFT order $a$ is shown for an example signal. The minimum TBP is attained when the signal support lies along the time or frequency axes with the corresponding FrFT orders 0 and \pm 1 . Therefore, TBP, which is the area of the dashed rectangle shown in (c), is not usually a tight measure for the time-frequency support of signals. As illustrated in (d), the GTBP is the area of the tightest bounding rectangle to the support of the signal in the time-frequency domain. Thus, it is a better measure for the time-frequency domain signal supports.

By using the order additivity and order periodicity property of the FrFT, it can be easily shown that the GTBP of a signal $x(t)$ and its $a_{0}$ th-order FrFT $x_{a_{0}}(t)$ is the same for any $a_{0}$.

As it has been investigated for the TBP, it is important to obtain the optimal STFT kernel, considering the GTBP of a signal. Actually, this investigation is relatively straightforward and can be conducted as follows. As shown in Fig. 2(b), for a signal $x(t)$ whose bounding rectangle is oriented at an angle $\phi_{0}$, which is not equal to 0 or $\pi / 2$, the fractionally Fourier transformed signal $x_{a_{0}}(t)$, where $a_{0}=(2 / \pi) \phi_{0}$, has its bounding rectangle oriented along the time axis. The transformed signal $x_{a_{0}}(t)$ has its TBP and GTBP equal to each other. Therefore, for $x_{a_{0}}(t)$, the optimal STFT window is the Gaussian window given in (26) with $T_{x_{a_{0}}}$ and $B_{x_{a_{0}}}$ as the corresponding time and bandwidth of the transformed signal. The desired time-frequency representation of $x(t)$ can be obtained as the counter-clockwise rotation of the optimal STFT for $x_{a_{0}}(t)$ by an angle of $\phi_{0}$. Since, as in (14)

$$
\begin{aligned}
& \operatorname{STFT}_{x_{a_{0}}}(t, f) \\
& \quad=\int x(\tau)\left[\int h\left(t^{\prime}-t\right) e^{\jmath 2 \pi f t^{\prime}} B_{-a_{0}}\left(t^{\prime}, \tau\right) d t^{\prime}\right]^{*} d \tau
\end{aligned}
$$

with optimal Gaussian kernel $h(t)=e^{-\pi \gamma t^{2}}$, where $\gamma=$ $B_{x_{a_{0}}} / T_{x_{a_{0}}}$, then the desired representation of $x(t)$ can be written as

$$
\begin{aligned}
D_{x}(t, f)= & R_{\phi_{0}}\left\{\operatorname{STFT}_{x_{a_{0}}}(t, f)\right\} \\
= & \int x(\tau) R_{\phi_{0}} \\
& \cdot\left\{\int h\left(t^{\prime}-t\right) e^{\jmath 2 \pi f t^{\prime}} B_{-a_{0}}\left(t^{\prime}, \tau\right) d t^{\prime}\right\}^{*} d \tau .
\end{aligned}
$$


In (29b), the expression in the brackets can be recognized as the $-a_{0}$ th-order FrFT of $h\left(t^{\prime}-t\right) e^{j 2 \pi f t^{\prime}}$, which is simply the time and frequency shifted form of the kernel $h(t)$. Using the time and frequency shift properties of the $\mathrm{FrFT}, \mathrm{D}_{x}(t, f)$ is computed as

$$
D_{x}(t, f)=e^{-\jmath \pi \psi} \int x(\tau) g_{\mathrm{GTBP}}^{*}(\tau-t) e^{-\jmath 2 \pi f \tau} d \tau
$$

where $\psi=\left(t^{2}-f^{2}\right) \sin \phi_{0} \cos \phi_{0}+2 t f \sin ^{2} \phi_{0}$, and the optimal kernel is

$$
\begin{array}{r}
g_{\mathrm{GTBP}}(\tau)=K e^{-\jmath \pi \tau^{2}\left(\cot \phi_{0}\left(\gamma^{2}-1\right)\right) /\left(\gamma^{2}+\cot ^{2} \phi_{0}\right)} \\
\cdot e^{-\pi \tau^{2}\left(\gamma \csc ^{2} \phi_{0}\right) /\left(\gamma^{2}+\cot ^{2} \phi_{0}\right)}
\end{array}
$$

where $K=\sqrt{\left(1+\jmath \cot \phi_{0}\right) /\left(\gamma+\jmath \cot \phi_{0}\right)}$. Since the phase $\psi$ can be ignored in $D_{x}(t, f)$, it is easy to see that the desired representation in (30) has the form of STFT with kernel $g_{\text {GTBP }}(\tau)$. This explicit form of the GTBP optimal STFT distribution provides significant computational saving in practice. Once the fractional order $a_{0}$ is determined, the computational complexity of (30) is the same as the computational complexity of the ordinary STFT. The discrete STFT is defined as [1]

$$
\operatorname{STFT}_{x}(n T, m F)=\int x\left(t^{\prime}\right) h^{*}\left(t^{\prime}-n T\right) e^{-\jmath 2 \pi m F t^{\prime}} d t^{\prime}
$$

where $n$ and $m$ are integers, and $T$ and $F$ are the sampling intervals of time and frequency, and it can be efficiently implemented by using FFT techniques.

There are many alternative approaches to determine the optimal fractional order $a_{0}$ to be used in the GTBP optimal STFT analysis. Since the optimal fractional order $a_{0}$ corresponding to a signal and its orientation in the time-frequency plane are related, the optimal order can be estimated by determining the orientation of the signal in the time-frequency plane. One way of determining the orientation of a signal in the time-frequency plane is to search for the peaks in the FrFT magnitudes computed at various fractional orders [14], [32], [33]. In practice, the search for the optimal fractional order can be conducted approximately by computing the FrFT of the signal at ten to 30 different fractional orders. Since each FrFT computation is $O(N \log N)$, the overall complexity of the required search is of $O(N \log N)$ as well. The FrFT computation algorithm is given in Table I [23], [27]. Alternatively, for mono-component signals, the required fractional Fourier transformation order can also be found based on the fractional moments of the signal. Efficient ways of computing the fractional Fourier transform moments of a signal are given in [34] and [35]. In addition, for signals with strong harmonics, an alternative mean of determining the required fractional Fourier transformation order can be found in
[36]. In practical applications, both the orientation angle of the signal and parameters related to the dimensions of the bounding rectangle in the appropriate fractional Fourier domain should be adaptively chosen. As future work, the performances of alternative ways of determining these parameters can be compared.

In Fig. 3, time-frequency domain localization by TBP optimal STFT and GTBP optimal STFT of a quadratic FM signal $x(t)=e^{3 \pi\left(t^{2}+0.1[t+0.5]^{3}\right)} e^{-\pi t^{2} / 9}$ shown in Fig. 3(a) is compared. The STFTs are evaluated with the kernels determined by using (26) and (31), as shown in Fig. 3(c) and (d), respectively. Time-frequency support of the GTBP optimal STFT illustrated in Fig. 3(f) is significantly better localized when compared with the TBP optimal STFT illustrated in Fig. 3(e).

The GTBP optimal STFT in (30) does not possess the rotation property because $g_{\mathrm{GTBP}}(\tau)$ is not a member of the Hermite-Gaussian function family; however, it has an envelope that is a scaled zeroth-order Hermite-Gaussian function. If the scaling is not equal to 1 , the rotation property cannot be satisfied. In the next section, we will provide a canonical decomposition of GTBP optimal STFT such that the rotation invariance is satisfied in a certain scaled fractional Fourier domain.

\section{GTBP OPTIMAL STFT AND ROTATION PROPERTY}

In Section IV, we have demonstrated that GTBP optimal STFT can be evaluated as efficiently as ordinary STFT. Using (30) and (31), the GTBP optimal STFT of a signal $x(t)$ with a support oriented at an angle of $\phi_{0}$ with respect to the time axis in the time-frequency plane is as in (33), shown at the bottom of the page.

Although (33) provides an efficient implementation method for the GTBP optimal STFT analysis, we would like to introduce a multistage implementation of it, as shown in Fig. 4(b), with its explicit derivation detailed in Appendix B. This decomposition is a linear canonical representation of the GTBP optimal STFT analysis with a sequence of operations explained and illustrated in Fig. 5. First, the $a_{0}$ th-order FrFT of the input signal is computed using (9). As shown in Fig. 5(d), the time-frequency support of the transformed signal [which is shown in Fig. 5(c)] is oriented along the time axis.

After this operation, the major axis of the time-frequency domain support of the transformed signal is along the time axis. Hence, the conventional TBP provides a good support information on the transformed signal. Assuming that the time and frequency widths of the transformed signal are $T_{x}$ and $B_{x}$, respectively, the time scaling of the transformed signal by an amount of $M=\sqrt{B_{x} / T_{x}}$ results in a signal $x(M t)$ whose time and frequency widths are equal. Hence, after the scaling, the time-frequency support will fit into a circular support. As shown in Fig. 5(f), the time-frequency support of the scaled signal [shown in Fig. 5(e)] fits into a circular region. Once the support of the

$$
\operatorname{STFT}_{x}(t, f)=K \int x(\tau) e^{-\pi(\tau-t)^{2}\left[\left(\left(\gamma \csc ^{2} \phi_{0}\right) /\left(\gamma^{2}+\cot ^{2} \phi_{0}\right)\right)-\jmath\left(\left(\cot \phi_{0}\left(\gamma^{2}-1\right)\right) /\left(\gamma^{2}+\cot ^{2} \phi_{0}\right)\right)\right]} e^{-\jmath 2 \pi f \tau} d \tau
$$


TABLE I

FAST Fractional Fourier Transform Algorithm Proposed IN [23]

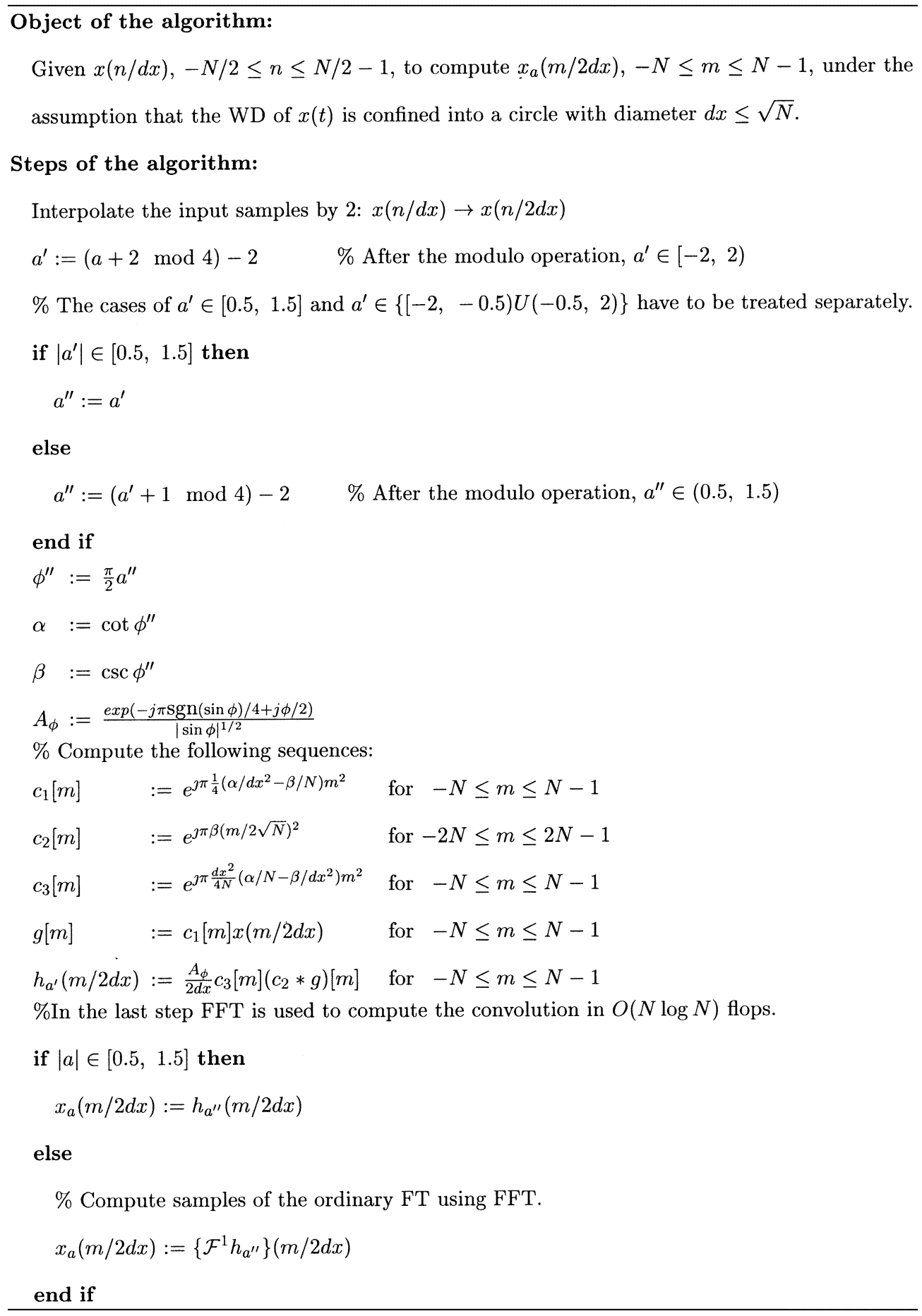

signal becomes circular, the GTBP optimal STFT and the TBP optimal STFT becomes identical. Hence, as it can be shown easily by using (26), the kernel of the GTBP optimal STFT is the following zeroth-order Hermite-Gaussian function

$$
g_{\mathrm{GTBP}}(t)=e^{-\pi t^{2}}
$$

The obtained STFT representation with the zeroth-order Hermite-Gaussian kernel should be operated in two successive stages to provide the final answer. First, a time-frequency domain inverse scaling should be performed:

$$
D_{s}(t, f)=D\left(\frac{t}{M}, M f\right)
$$

where $D(t, f)$ is the input, and $D_{s}(t, f)$ is the output of the timefrequency inverse scaling operation. The effect of this scaling operation is shown in Fig. 5(f) and (g). Then, the final GTBP 

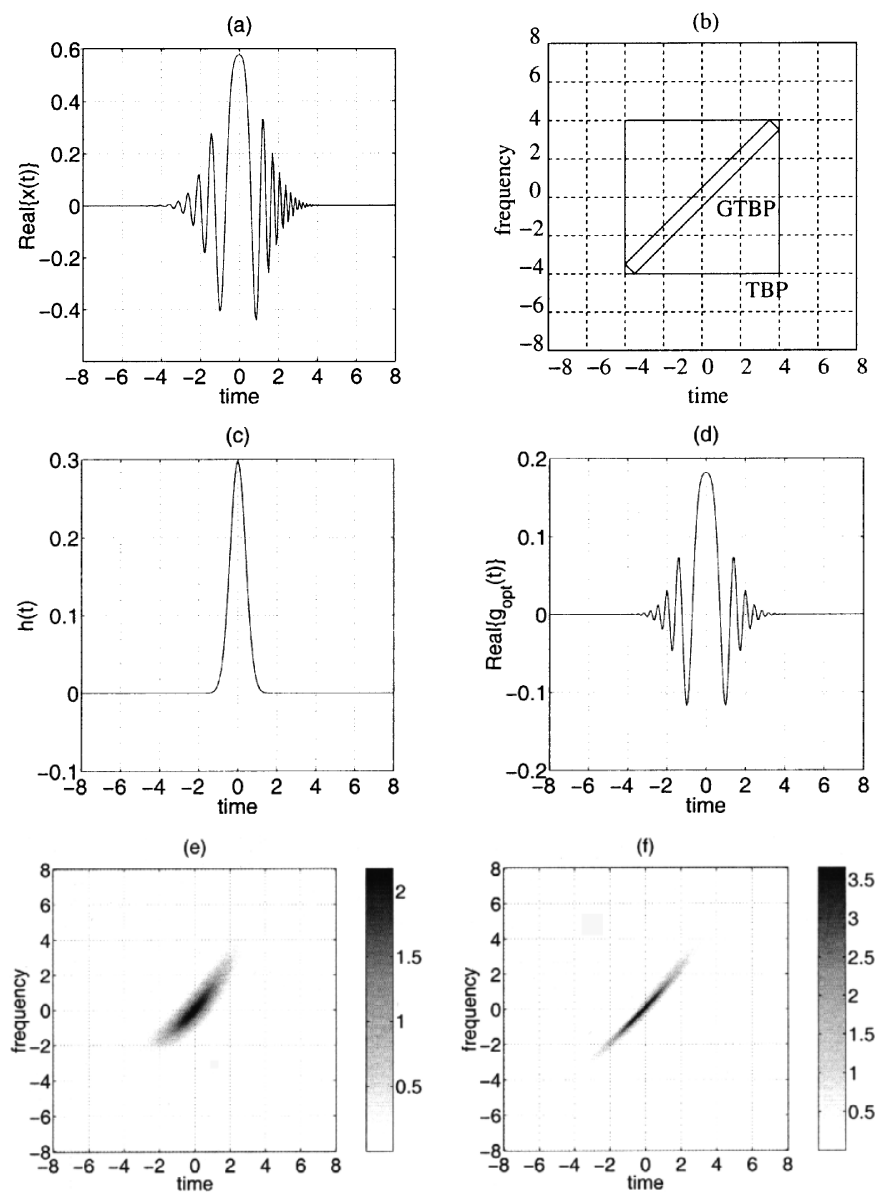

Fig. 3. Time-frequency domain localization by the TBP and GTBP optimal STFT of a quadratic FM signal $x(t)=e^{j \pi\left(t^{2}+0.1[t+0.5]^{3}\right)} e^{-\pi t^{2} / 9}$ shown in (a) is compared. The rectangles whose area are equal to the TBP and GTBP, respectively, are illustrated in (b). The TBP optimal STFT is evaluated with the kernel shown in (c), and the GTBP optimal STFT is evaluated with the kernel shown in (d). The GTBP optimal STFT illustrated in (f) has a significantly improved time-frequency support than the TBP optimal STFT illustrated in (e).

optimal STFT distribution is obtained by the following rotation operation:

$$
\operatorname{STFT}_{x}(t, f)=R_{\phi}\left\{D_{s}(t, f)\right\} .
$$

The effect of this operation is shown in Fig. 5(h), which yields a high-resolution time-frequency description corresponding to the original signal.

The main reason behind the introduction of this canonical decomposition is that the rotation invariant STFT with the zeroth-order Hermite-Gaussian kernel is explicitly shown to be part of every GTBP optimal STFT analysis. Therefore, for any arbitrary mono-component signal, there exists a "natural domain," where the rotation independent STFT analysis with zeroth-order Hermite-Gaussian kernel provides the GTBP optimal STFT representation. The signals are transformed to their "natural domains" by the first two operations of the canonical decomposition. We believe this concept of "natural domain" is theoretically significant and will provide further insight to the research on time-frequency signal analysis.

In the rest of this section, the performance of the GTBP optimal STFT is illustrated by using simulated data. In the simu- lations, we use a quadratic FM signal embedded in $-5 \mathrm{~dB}$ noise. The analyzed signal is $x(t)=(1 / \sqrt{3}) e^{j \pi\left[\alpha t^{2}+\beta(t-\eta)^{3}\right]} e^{-\pi \gamma t^{2}}$, where $\alpha=1, \beta=0.2, \eta=1.5$, and $\gamma=1 / 18$. The time domain signal and the corresponding TBP optimal STFT are shown in Fig. 6(a) and (b). In Fig. 6(c), the peak amplitudes of the fractional Fourier transformed $x(t)$ as a function of $\phi-\pi / 2$ is presented. The peak is observed at the angle $\phi_{0}=65^{\circ}$. Finally, the GTBP optimal STFT is shown in Fig. 6(d).

A significant improvement for the time-frequency localization is observed when compared with the TBP optimal STFT with similar computational complexity. As future work, optimal STFT analysis will be extended to multicomponent signals. To obtain a well-localized time-frequency representation of a multicomponent chirp signal with different chirp rates, the orientation angles of each component and, consequently, the required FrFT orders to perform the STFT, should be determined. Following the individual GTBP optimal time-frequency analysis of each signal component, the obtained time-frequency representations are combined so that the time-frequency localization of each chirp component is optimally compact.

\section{CONClusions AND Future DiRections}

In this paper, we have investigated the magnitude-wise shift invariance and time-frequency rotation properties of linear time-frequency representations that are satisfied by the quadratic WD. It is proven that STFT is the only linear distribution that is magnitude-wise shift invariant in both time and frequency. Furthermore, it is shown that the STFT satisfies the rotation property if its kernel is chosen as a Hermite-Gaussian function.

We investigated TBP optimal STFT analysis. By generalizing the TBP to fractional Fourier domains, the GTBP definition is introduced. It is shown that the GTBP provides a rotation invariant measure for the time-frequency support of mono-component signals. Then, the GTBP optimal STFT analysis is proposed for mono-component signals.

Along with the proposed efficient implementation of the GTBP optimal STFT, a theoretically insightful canonical decomposition of it is presented. This way, the GTBP optimal STFT analysis is related to the rotationally invariant STFT analysis with the zeroth-order Hermite-Gaussian kernel. In addition, this decomposition provided a "natural domain" concept for mono-component signals.

The proposed GTBP optimal STFT requires three important parameters related to the time-frequency support of mono-component signals. These are the dimensions and the orientation of the bounding rectangle [37]. In practical applications, these parameters should be adaptively chosen. Comparison of the performances of alternative ways of determining these parameters requires further investigation.

In addition, the GTBP optimal STFT representation of multicomponent signals needs the determination of the required set of parameters for each signal component. Efficient ways of their determination and how the individual GTBP optimal STFT representations should be combined require further research. In addition, further research is required in obtaining other forms of 


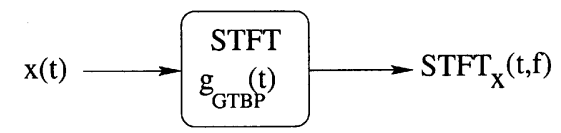

(a)

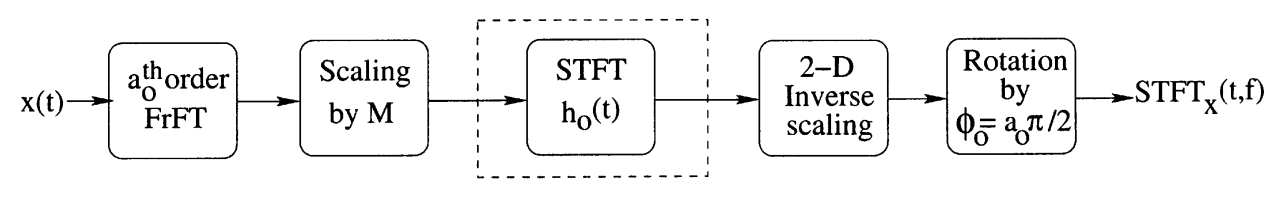

(b)

Fig. 4. (a) Block diagram of GTBP optimal STFT. (b) Linear canonical decomposition of it. The first two operational blocks in (b) transform the mono-component signal $x(t)$ to its "natural domain," where the rotation invariant STFT with kernel $h_{0}(t)$ provides the GTBP optimal STFT distribution.
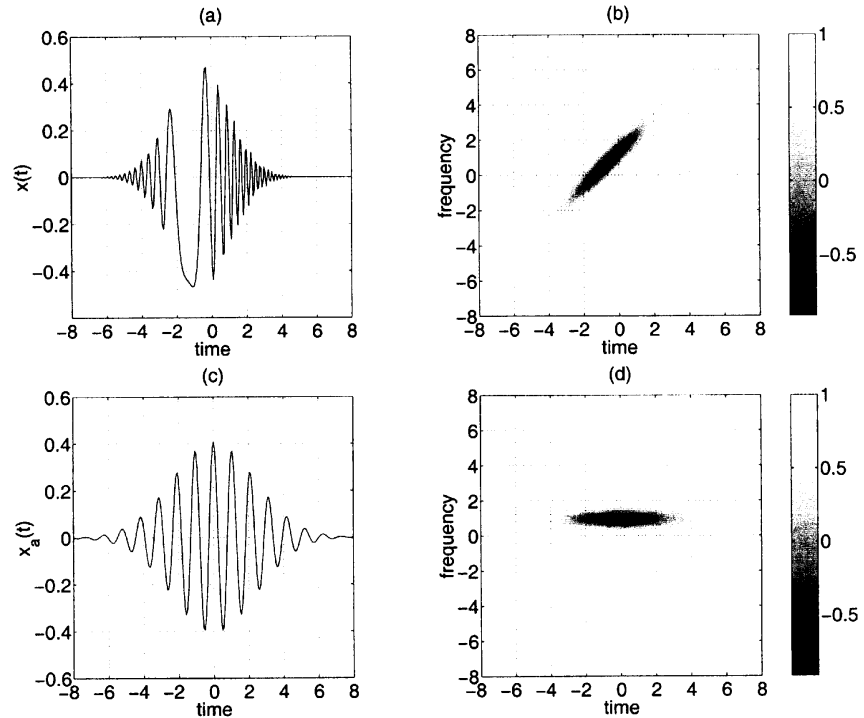

(e)

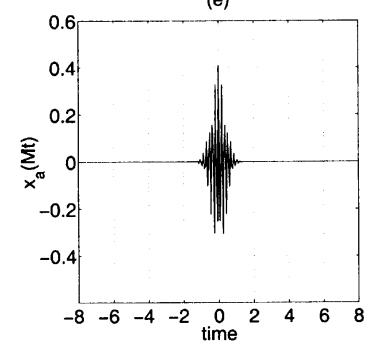

(g)
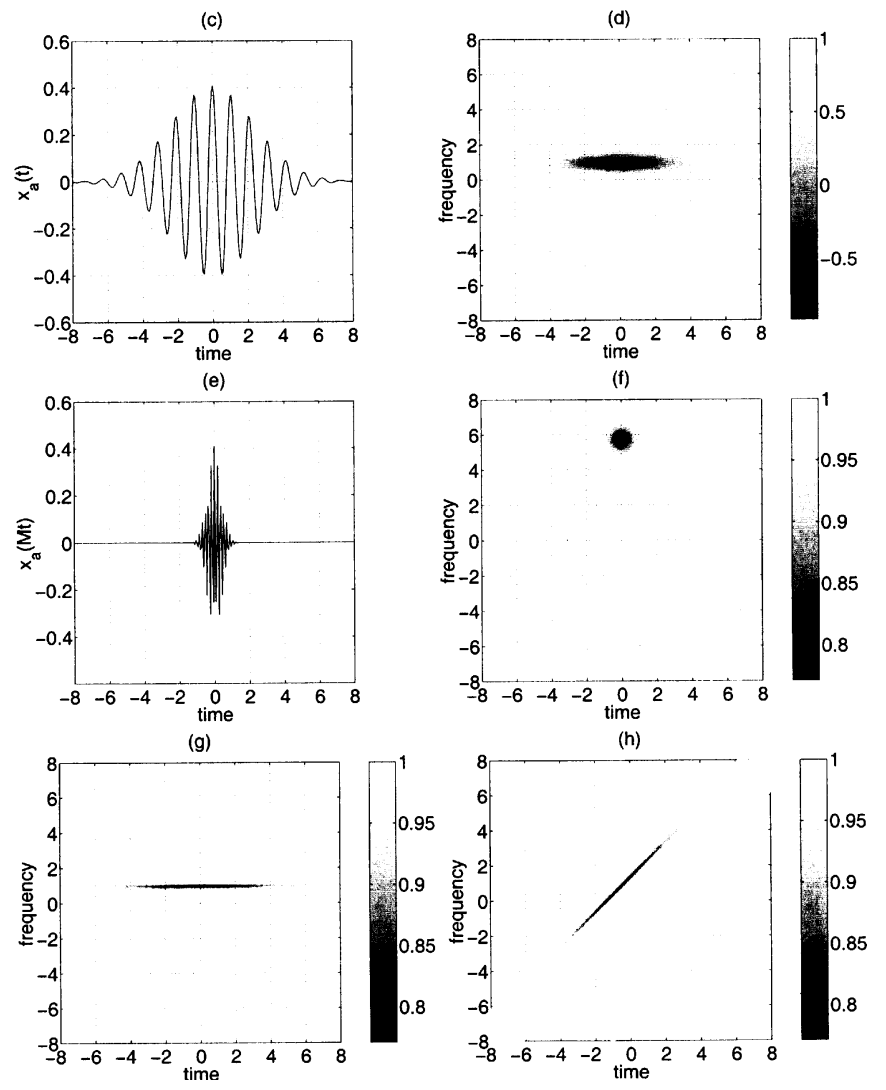

Fig. 5. For a chirp-like signal shown in (a), the FrFT is computed so that the chirp is converted to a sinusoidal, as in (c). The corresponding STFTs are shown in (b) and (d), respectively. Through appropriate scaling $x_{a}(t)$ is converted to a zeroth-order Hermite-Gaussian enveloped sinusoidal, as illustrated in (e), and its STFT is computed with the Gaussian window, as shown in (f). This is followed by 2-D scaling, which inverts the scaling on the signal as shown in (g). Finally, the distribution is rotated back to its original orientation removing the FrFT effect, as illustrated in (h). generalized time-bandwidth products that are invariant under a more general area preserving time-frequency operations: the symplectic transforms [38].

\section{APPENDIX A}

\section{Theorem 1}

If a linear system $\mathcal{T}$ satisfies magnitude-wise shift invariance in time, then there exist an $h(t)$ and $\widehat{\phi}(t)$ such that the output of $\mathcal{T}$ for any arbitrary input $x(t)$ can be written as

$$
\mathcal{T}\{x(t)\}=e^{\widehat{\jmath}(t)}[h(t) * x(t)] .
$$

Proof: By using the Riesz theorem, $\mathcal{T}$ can be represented as

$$
\mathcal{T}\{x(t)\}=\int K\left(t, t^{\prime}\right) x\left(t^{\prime}\right) d t^{\prime}
$$

where $K\left(t, t^{\prime}\right)$ is the kernel of the transformation. If $\mathcal{T}$ satisfies magnitude-wise shift invariance in time, the outputs to impulses $\delta(t)$ and $\delta\left(t-t_{s}\right), y(t)$ and $y_{s}(t)$, respectively, should satisfy $\left|y_{s}(t)\right|=\left|y\left(t-t_{s}\right)\right|$, which implies that

$$
\left|K\left(t-t_{s}, 0\right)\right|=\left|K\left(t, t_{s}\right)\right|, \quad \forall t, t_{s}
$$

In general, the kernel function can be decomposed as $K\left(t, t^{\prime}\right)=$ $\varrho\left(t, t^{\prime}\right) e^{\jmath \phi\left(t, t^{\prime}\right)}$, where $\varrho\left(t, t^{\prime}\right)$ and $\phi\left(t, t^{\prime}\right)$ are the magnitude and phase functions, respectively. The condition in (39) requires that $\varrho\left(t, t^{\prime}\right)=\varrho\left(t-t^{\prime}\right)$; therefore, the kernel function can be decomposed as

$$
K\left(t, t^{\prime}\right)=\varrho\left(t-t^{\prime}\right) e^{\jmath \phi\left(t, t^{\prime}\right)} .
$$

Next, it will be shown that the phase function satisfies

$$
\phi\left(t, t^{\prime}\right)=-\Psi\left(t-t^{\prime}\right)+\widehat{\phi}(t) .
$$

To prove (41), the input can be chosen as a linear combination of two weighted impulses $x(t)=\alpha_{1} \delta(t)+\alpha_{2} \delta(t-\tau)$; then, the 

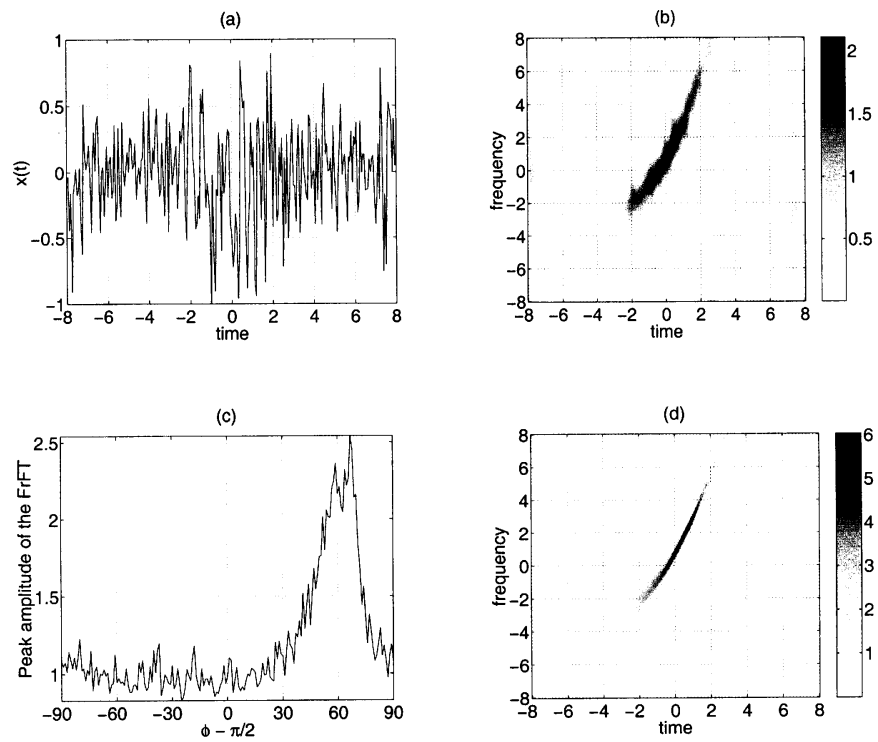

Fig. 6. To illustrate the effect of noise, the noisy quadratic FM signal shown in (a) is analyzed by both the TBP optimal STFT and the GTBP optimal STFT. The corresponding TBP optimal STFT is shown in (b). The optimum fractional Fourier order for the signal can be identified automatically by using the peak amplitudes of the FrFT magnitude data as a function of the orientation angle, as shown in (c). The observed peak at the angle $\phi_{0}=65^{\circ}$ is used in the GTBP optimal STFT distribution shown in (d).

output is $y(t)=\alpha_{1} K(t, 0)+\alpha_{2} K(t, \tau)$. For the shifted input $x_{s}(t)=x\left(t-t_{s}\right)$, the output becomes $y_{s}(t)=\alpha_{1} K\left(t, t_{s}\right)+$ $\alpha_{2} K\left(t, t_{s}+\tau\right)$. The magnitude-wise shift invariance implies $\left|y_{s}(t)\right|=\left|y\left(t-t_{s}\right)\right|$. Thus, the kernel should satisfy

$\left|\alpha_{1} K\left(t, t_{s}\right)+\alpha_{2} K\left(t, t_{s}+\tau\right)\right|$

$$
=\left|\alpha_{1} K\left(t-t_{s}, 0\right)+\alpha_{2} K\left(t-t_{s}, \tau\right)\right|
$$

for all $t, t_{s}, \tau, \alpha_{1}, \alpha_{2}$. Using the definition in (40), (42) can be re-expressed as

$$
\begin{aligned}
& \left|\alpha_{1} \varrho\left(t-t_{s}\right) e^{\jmath \phi\left(t, t_{s}\right)}+\alpha_{2} \varrho\left(t-t_{s}-\tau\right) e^{\jmath \phi\left(t, t_{s}+\tau\right)}\right| \\
& \quad=\left|\alpha_{1} \varrho\left(t-t_{s}\right) e^{\jmath \phi\left(t-t_{s}, 0\right)}+\alpha_{2} \varrho\left(t-t_{s}-\tau\right) e^{\jmath \phi\left(t-t_{s}, \tau\right)}\right| .
\end{aligned}
$$

Assuming that $\varrho(t)$ is not identically zero, (43) can only be satisfied if

$$
\begin{array}{r}
\phi\left(t, t_{s}\right)-\phi\left(t-t_{s}, 0\right) \\
\quad=\phi\left(t, t_{s}+\tau\right)-\phi\left(t-t_{s}, \tau\right), \forall t, t_{s}, \tau .
\end{array}
$$

After rearranging the terms in (44), we obtain the following condition:

$$
\begin{aligned}
\phi\left(t, t_{s}+\tau\right)- & \phi\left(t, t_{s}\right) \\
& =\phi\left(t-t_{s}, \tau\right)-\phi\left(t-t_{s}, 0\right), \forall t, t_{s}, \tau .
\end{aligned}
$$

It can be shown that if $\phi\left(t, t^{\prime}\right)$ satisfies (45), then $\psi\left(t, t^{\prime}\right)=$ $\left(\partial / \partial t^{\prime}\right) \phi\left(t, t^{\prime}\right)$ exists. Thus, in the limit $\tau$ approaching 0 , (45) implies that

$$
\psi\left(t, t_{s}\right)=\psi\left(t-t_{s}, 0\right)=\psi\left(t-t_{s}\right), \forall t, t_{s} .
$$

Therefore, $\phi\left(t, t^{\prime}\right)$ satisfies the following partial differential equation:

$$
\frac{\partial}{\partial t^{\prime}} \phi\left(t, t^{\prime}\right)=\psi\left(t-t^{\prime}\right)
$$

which is solved by

$$
\phi\left(t, t^{\prime}\right)=-\Psi\left(t-t^{\prime}\right)+\widehat{\phi}(t)
$$

where $\psi(t)=(d / d t) \Psi(t)$, and $\widehat{\phi}(t)$ is an arbitrary phase function. Thus, the kernel has the following form:

$$
K\left(t, t^{\prime}\right)=\varrho\left(t-t^{\prime}\right) e^{-\jmath \Psi\left(t-t^{\prime}\right)} e^{\widehat{\jmath}(t)} .
$$

Hence, the input-output relationship of the linear system can be written as

$$
\begin{aligned}
y(t) & =\int K\left(t, t^{\prime}\right) x\left(t^{\prime}\right) d t^{\prime} \\
& =\int \varrho\left(t-t^{\prime}\right) e^{-\jmath \Psi\left(t-t^{\prime}\right)} e^{\widehat{\jmath \phi}(t)} x\left(t^{\prime}\right) d t^{\prime} \\
& =e^{\widehat{\jmath}(t)}[h(t) * x(t)]
\end{aligned}
$$

where $h(t)=\varrho(t) e^{-\jmath \Psi(t)}$.

\section{APPENDIX B}

In this Appendix, we prove that the linear canonical decomposition of the GTBP optimal STFT described in Section V and its computationally efficient form in (33) are explicitly equivalent.

The multistage implementation of the GTBP optimal STFT analysis shown in Fig. 4(b) starts with the FrFT operation at the optimum fractional order $a_{0}$ and scaling the signal by $M=\sqrt{T_{x_{a_{0}}} / B_{x_{a_{0}}}}$ so that $x_{a_{0}}(M t)$ is obtained. The rotation-invariant STFT of $x_{a_{0}}(M t)$ with the kernel of the zeroth-order Hermite-Gaussian function is computed as

$$
\operatorname{STFT}_{x_{a_{0}}(M t)}(t, f)=\int x_{a_{0}}(M \tau) h_{0}^{*}(\tau-t) e^{-j 2 \pi f \tau} d \tau .
$$

The STFT operation is followed by a 2-D time-frequency domain scaling through transforming time and frequency variables $(t, f)$ to $(t / M, M f)$ so that the resultant distribution $D_{s}(t, f)$ is

$$
D_{s}(t, f)=\int x_{a_{0}}(M \tau) h_{0}^{*}\left(\tau-\frac{t}{M}\right) e^{-\jmath 2 \pi M f \tau} d \tau .
$$

Using the definition in (9) and the property $B_{a_{0}}\left(t^{\prime}, \tau\right)=$ $B_{-a_{0}}^{*}\left(t^{\prime}, \tau\right)$ of the FrFT kernel, $D_{s}(t, f)$ can be re-expressed as

$$
\begin{aligned}
D_{s}(t, f) & =\frac{1}{M} \int x(\tau) \\
\cdot & {\left[\int B_{-a_{0}}\left(\tau, \tau^{\prime}\right) h_{0}\left(\frac{\tau^{\prime}-t}{M}\right) e^{\jmath 2 \pi f \tau^{\prime}} d \tau^{\prime}\right]^{*} d \tau . }
\end{aligned}
$$

The expression in the square brackets in (53) is the $-a_{0}$ th-order FrFT of the scaled, time and frequency shifted zeroth-order Her- 
mite-Gaussian function $f(\tau)=h_{0}((\tau-t) / M) e^{\jmath 2 \pi f \tau}$. Using the FrFT properties in [21], $f_{-a_{0}}(\tau)$ can be expressed as

$$
\begin{aligned}
f_{-a_{0}}(\tau) & \\
= & M K e^{-\jmath \pi \psi} h_{0}\left(\frac{M \sin \phi_{0}^{\prime}}{\sin \phi_{0}}\left(\tau+f \sin \phi_{0}-t \cos \phi_{0}\right)\right) \\
& \cdot e^{\jmath 2 \pi \tau\left(f \cos \phi_{0}+t \sin \phi_{0}\right)} \\
& \times e^{-\jmath \pi\left(\tau+f \sin \phi_{0}-t \cos \phi_{0}\right)^{2} \cot \phi_{0}\left(1-\left(\cos ^{2} \phi^{\prime} / \cos ^{2} \phi\right)\right)}
\end{aligned}
$$

where $\phi_{0}=a_{0} \pi / 2, K=\sqrt{\left(1+\jmath \cot \phi_{0}\right) /\left(1 / M^{2}+\jmath \cot \phi_{0}\right)}$, $\psi=\sin \phi_{0} \cos \phi_{0}\left(t^{2}-f^{2}\right)+2 t f \sin ^{2} \phi_{0}$, and $\phi_{0}^{\prime}=$ $\tan ^{-1}\left(\tan \phi_{0} / M^{2}\right)$.

Finally, $D_{s}(t, f)$ is rotated by $\phi_{0}=a_{0} \pi / 2$ in the counter clockwise direction, and $R_{\phi_{0}}\left\{D_{s}(t, f)\right\}$ is obtained as

$$
\begin{aligned}
& R_{\phi_{0}}\left\{D_{s}(t, f)\right\} \\
& =D_{s}\left(t \cos \phi_{0}+f \sin \phi_{0},-t \sin \phi_{0}+f \cos \phi_{0}\right) \\
& =e^{-\jmath \pi \psi_{r}} \int x(\tau) K h_{0}^{*}\left(\frac{M \sin \phi_{0}^{\prime}}{\sin \phi_{0}}[\tau-t]\right) \\
& \quad \cdot e^{-\jmath \pi \cot \phi_{0}\left(1-\cos ^{2} \phi_{0}^{\prime} / \cos ^{2} \phi_{0}\right)(\tau-t)^{2}} e^{-\jmath 2 \pi f \tau} d \tau
\end{aligned}
$$

where

$$
\begin{aligned}
\psi_{r}=\left(t^{2}-f^{2}\right)\left(\sin \frac{4 \phi_{0}}{4}\right. & \left.-\sin 2 \phi_{0} \sin ^{2} \phi_{0}\right) \\
& +t f\left(2 \cos 2 \phi_{0} \sin ^{2} \phi_{0}+\sin ^{2} 2 \phi_{0}\right) .
\end{aligned}
$$

Since the phase $\psi_{r}$ can be ignored, it is simply seen that (56) is an STFT with the kernel function

$$
\begin{aligned}
g(\tau)= & K h_{0}^{*}\left(\frac{M \sin \phi_{0}^{\prime}}{\sin \phi_{0}} \tau\right) e^{\jmath \pi \tau^{2} \cot \phi_{0}\left(1-\cos ^{2} \phi_{0}^{\prime} / \cos ^{2} \phi_{0}\right)} \\
= & K e^{-\pi\left(\left(M^{2} \sin ^{2} \phi_{0}^{\prime}\right) /\left(\sin ^{2} \phi_{0}\right)\right) \tau^{2}} \\
& \cdot e^{\jmath \pi \tau^{2} \cot \phi_{0}\left(1-\cos ^{2} \phi_{0}^{\prime} / \cos ^{2} \phi_{0}\right)} .
\end{aligned}
$$

Since $M$ in (58) and $\gamma$ in (31) are related as $\gamma=1 / M^{2}$, and $\sin \phi_{0}^{\prime}$ and $\cos \phi_{0}^{\prime}$ are

$$
\begin{aligned}
\sin \phi_{0}^{\prime} & =\frac{\tan \phi_{0}}{\sqrt{\tan ^{2} \phi_{0}+M^{4}}} \\
\cos \phi_{0}^{\prime} & =\frac{M^{2}}{\sqrt{\tan ^{2} \phi_{0}+M^{4}}}
\end{aligned}
$$

(58) can be re-expressed as

$$
\begin{array}{r}
g(\tau)=K e^{-\jmath \pi \tau^{2}\left(\cot \phi_{0}\left(\gamma^{2}-1\right)\right) /\left(\gamma^{2}+\cot ^{2} \phi_{0}\right)} \\
\cdot e^{-\pi \tau^{2}\left(\gamma \csc ^{2} \phi_{0}\right) /\left(\gamma^{2}+\cot ^{2} \phi_{0}\right)}
\end{array}
$$

which equals the kernel function $g_{\mathrm{GTBP}}(\tau)$ in (31).

\section{ACKNOWLEDGMENT}

The authors would like to thank A. K. Özdemir for many useful discussions and insightful comments.

\section{REFERENCES}

[1] F. Hlawatsch and G. F. Boudreaux-Bartels, "Linear and quadratic timefrequency signal representations," IEEE Signal Processing Mag., vol. 9, pp. 21-67, Apr. 1992.

[2] L. Cohen, "Time-frequency distributions-A review," Proc. IEEE, vol. 77, pp. 941-981, July 1989.

[3] - Time-Frequency Analysis. Englewood Cliffs, NJ: Prentice-Hall, 1995.

[4] R. A. Altes, "Detection, estimation and classification with spectrograms," J. Acoust. Soc. Amer, vol. 67, no. 4, pp. 1232-1246, Apr. 1980.

[5] S. Cheung and J. S. Lim, "Combined multiresolution (wideband/narrow-band) spectrogram," IEEE Trans. Signal Processing, vol. 40, pp. 975-977, Apr. 1992.

[6] E. J. Rothwell, K. M. Chen, and D. P. Nyquist, "An adaptive-windowwidth short-time Fourier transform for visualization of radar target substructure resonances," IEEE Trans. Antennas Propagat., vol. 46, pp. 1393-1395, Sept. 1998.

[7] H. K. Kwok and D. L. Jones, "Improved instantaneous frequency estimation using an adaptive short-time Fourier transform," IEEE Trans. Signal Processing, vol. 48, pp. 2964-2972, Oct. 2000.

[8] LJ. Stankovic, "A method for time-frequency signal analysis," IEEE Trans. Signal Processing, vol. 42, pp. 225-229, Jan. 1994.

[9] P. K. Kumar and K. M. M. Prabhu, "Simulation studies of moving-target detection: A new approach with Wigner-Ville distribution," Proc. Inst. Elect. Eng. Radar. Sonar Navig., vol. 144, pp. 259-265, Oct. 1997.

[10] P. Flandrin, "Some features of time-frequency representations of multicomponent signals," in Proc. IEEE Int. Conf. Acoust. Speech Signal Process., vol. 9, 1984, pp. 266-269.

[11] K. J. R. Liu, "Novel parallel architecture for short-time Fourier transform," IEEE Trans. Circuits Syst., vol. 40, pp. 786-789, Dec. 1993.

[12] M. G. Amin and K. D. Feng, "Short-time Fourier transform using cascade filter structures," IEEE Trans. Circuits Syst., vol. 42, pp. 631-641, Oct. 1995

[13] W. Chen, N. Kehtarnavaz, and T. W. Spencer, "An efficient recursive algorithm for time-varying Fourier transform," IEEE Trans. Signal Processing, vol. 41, pp. 2488-2490, July 1993.

[14] A. W. Lohmann and B. H. Soffer, "Relationships between the RadonWigner and fractional Fourier transforms," J. Opt. Soc. Amer. A, vol. 11, pp. 1798-1801, 1994.

[15] J. C. Wood and D. T. Barry, "Radon transformation of time-frequency distributions for analysis of multicomponent signals," IEEE Trans. Signal Processing, vol. 42, pp. 3166-3177, Nov. 1994

[16] G. Jones and B. Boashash, "Generalized instantaneous parameters and window matching in the time-frequency plane," IEEE Trans. Signal Processing, vol. 45, pp. 1264-1275, May 1997.

[17] L. L. Scharf and B. Friedlander, "Toeplitz and Hankel kernels for estimating time-varying spectra of discrete-time random processes," IEEE Trans. Signal Processing, vol. 49, pp. 179-189, Jan. 2001.

[18] A. W. Naylor and G. R. Sell, Linear Operator Theory in Engineering and Science. New York: Springer-Verlag, 1982.

[19] L. B. Almedia, "The fractional Fourier transform and time-frequency representations," IEEE Trans. Signal Processing, vol. 42, pp. 3084-3091, Nov. 1994

[20] V. Namias, "The fractional order Fourier transform and its application to quantum mechanics," J. Inst. Math. Appl., vol. 25, pp. 241-265, 1980.

[21] H. M. Ozaktas, Z. Zalevsky, and M. A. Kutay, The Fractional Fourier Transform With Applications in Optics and Signal Processing. New York: Wiley, 2000.

[22] H. M. Ozaktas, N. Erkaya, and M. A. Kutay, "Effect of fractional Fourier transformation on time-frequency distributions belonging to Cohen class," IEEE Signal Processing Lett., vol. 3, pp. 40-41, Feb. 1996.

[23] H. M. Ozaktas, O. Arıkan, M. A. Kutay, and G. Bozdagi, "Digital computation of the fractional Fourier transform," IEEE Trans. Signal Processing, vol. 44, pp. 2141-2150, Sept. 1996.

[24] J. C. Wood and D. T. Barry, "Tomographic time-frequency analysis and its application toward time-varying filtering and adaptive kernel desing for multicomponent linear-FM signals," IEEE Trans. Signal Processing, vol. 42, pp. 2094-2104, Aug. 1994.

[25] — , "Linear signal synthesis using the Radon-Wigner transform," IEEE Trans. Signal Processing, vol. 42, pp. 2105-2111, Aug. 1994.

[26] Z. Bao, G. Wang, and L. Luo, "Inverse synthetic aperture radar imaging of maneuvering targets," Opt. Eng., vol. 37, no. 5, pp. 1582-1588, May 1998 
[27] A. K. Özdemir and O. Arıkan, "Efficient computation of the ambiguity function and the Wigner distribution on arbitrary line segments," IEEE Trans. Signal Processing, vol. 49, pp. 381-393, Feb. 2001.

[28] H. Ozaktas and O. Aytür, "Fractional Fourier domains," Signal Process., vol. 46, pp. 119-124, 1995

[29] S. Shinde and V. M. Gadre, "An uncertainty principle for real signals in the fractional Fourier transform domain," IEEE Trans. Signal Processing, vol. 49, pp. 2545-2548, Nov. 2001.

[30] A. Bultan, "A Four-Parameter Atomic Decomposition and the Related Time-Frequency Distribution,” Ph.D. dissertation, Dept. Electr. Electron. Eng., Middle East Tech. Univ., Ankara, Turkey, 1995.

[31] _ - "A four-parameter atomic decomposition of chirplets," IEEE Trans. Signal Processing, vol. 47, pp. 731-745, Mar. 1999.

[32] S. Pei and M. Yeh, "A novel method for discrete fractional Fourier transform computation," in Proc. IEEE Int. Symp. Circuits Syst., vol. 2, 2001, pp. $585-588$.

[33] O. Akay and G. F. Boudreaux-Bartels, "Fractional autocorrelation and its application to detection and estimation of linear fm signals," in Proc. IEEE-SP Int. Symp. Time-Freq. Time-Scale Anal., 1998, pp. 213-216.

[34] T. Alieva and M. J. Bastiaans, "On fractional Fourier transform moments," IEEE Signal Processing Lett., vol. 7, pp. 320-323, Nov. 2000.

[35] L. Stankovic, T. Alieva, and M. Bastiaans, "Wigner distribution weighted in the fractional Fourier domains," in Proc. ProRISC, Veldhoven, The Netherlands, Nov. 2001, pp. 635-640.

[36] F. Zhang, Y. Q. Chen, and G. Bi, "Adaptive harmonic fractional Fourier transform," IEEE Signal Processing Lett., vol. 6, pp. 281-283, Nov. 1999.

[37] A. K. Özdemir, L. Durak, and O. Arıkan, "High resolution time-frequency analysis by fractional domain warping," in Proc. IEEE Int. Conf. Acoust., Speech, Signal Process., May 2001.

[38] A. J. E. M. Janssen, "On the locus and spread of pseudo-density functions in the time-frequency plane," Philips J. Res., vol. 37, pp. 79-110, 1982.

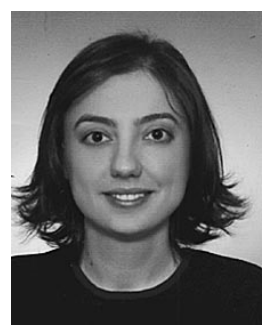

Lütfiye Durak (S'94) was born in 1974 in Sivas, Turkey. She received the B.Sc. and M.S. degrees in electrical and electronics engineering from Bilkent University, Ankara, Turkey, in 1996 and 1999, respectively. She is currently pursuing the Ph.D. degree under the supervision of O. Arıkan with the Department of Electrical and Electronics Engineering, Bilkent University.

During her M.S. and Ph.D. studies, she has been a teaching and research assistant. Her current research interests are in digital signal processing and its applications to time-frequency analysis.

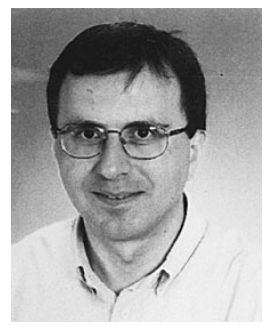

Orhan Arikan (M'91) was born in 1964 in Manisa, Turkey. He received the B.Sc. degree in electrical and electronics engineering from the Middle East Technical University, Ankara, Turkey, in 1986 and both the M.S. and Ph.D. degrees in electrical and computer engineering from the University of Illinois at Urbana-Champaign, in 1988 and 1990, respectively.

Following his graduate studies, he worked for three years as a Research Scientist at Schlumberger-Doll Research, Ridgefield, CT. During this time, he was involved in the inverse problems and fusion of measurements with multiple modality. He joined Bilkent University, Ankara, in 1993, where he is presently Associate Professor of electrical engineering. His current research interests are in adaptive signal processing, time-frequency analysis, inverse problems, and array signal processing. 